\title{
Zeno effect in degree of polarization of a single photon or quantum-state purity of a spin-1/2 system
}

\author{
Alfredo Luis and Isabel Gonzalo* \\ Departamento de Óptica, Facultad de Ciencias Físicas, Universidad Complutense, 28040 Madrid, Spain \\ Miguel A. Porras \\ Departamento de Física Aplicada a los Recursos Naturales and Grupo de Sistemas Complejos, Universidad Politécnica de Madrid, \\ Rios Rosas 21, 28003 Madrid, Spain
}

(Received 9 April 2013; revised manuscript received 14 May 2013; published 20 June 2013)

\begin{abstract}
We describe a version of the Zeno effect where the monitored physical property cannot be represented by a standard operator in the system space and the evolution to be prevented by observation is not unitary. This is the case of quantum-state purity of a spin- $1 / 2$ system, which is equivalent to the degree of polarization of a single photon. By a suitable embedding of the system (say, a single photon) in an enlarged space of a pair of photons, pure and fully mixed components lead to orthogonal subspaces, with lack of purity becoming equivalent to entanglement. By imposing desirable properties the representation of the system in the enlarged space is unique. We show that the Zeno effect is possible, and experimentally feasible, in the enlarged space. The peculiarity that photons in identical polarization states are involved in pairs to observe the dynamics of each photon allows us to refer it as the self-Zeno effect or Narcissus effect.
\end{abstract}

DOI: 10.1103/PhysRevA.87.064102

PACS number(s): 03.65.Xp, 42.25.Fx, 42.60.Da, 03.65.Ta

\section{INTRODUCTION}

The Zeno effect is a way to explore quantum and classical features related to observation effects [1]. In its most classic form, the Zeno effect is the alteration of unitary evolution when monitoring a physical property usually represented by an Hermitian operator. In this work we undertake a different version of the Zeno effect, where the evolution is not unitary and the monitored physical property is not represented by a Hermitian operator.

More specifically, our problem deals with the evolution and observation of the quantum-state purity of a spin- $1 / 2$ system. We study whether purity observation prevents purity evolution. For definiteness we focus on a quantum-optical realization where the effective spin- $1 / 2$ system is the transverse polarization of a single photon. In such a case the quantum-state purity is equivalent to the classic degree of polarization. In the classic program of the Zeno effect, the photon should experience a transformation that changes the degree of polarization and is frequently interrupted to observe its degree of polarization.

The main obstacle of this program is that there is no operator representing the degree of polarization in the Hilbert space $H_{s}$ of the single-system realization $\rho$. The usual measurement of the degree of polarization is an indirect one, through four detectors providing the Stokes parameters.

In order to avoid this obstacle we regard lack of quantumstate purity as an effect of entanglement in a larger system, so it is advantageous to embed the system in a larger space. To this end, the simplest choice is system realization in a doubled Hilbert space $H_{s} \otimes H_{s}$, grouping the photons in pairs, always with the same reduced state $\rho$ for each photon. The key point is that in the doubled space $H_{s} \otimes H_{s}$ the unpolarized component of $\rho$ can be well represented by the fully entangled state $|H\rangle_{1}|V\rangle_{2}-|V\rangle_{1}|H\rangle_{2}$ (with $H, V$ representing horizontal and vertical polarization for each

*Corresponding author: igonzalo@ fis.ucm.es photon), which is orthogonal to the state $|V\rangle_{1}|V\rangle_{2}$ or $|H\rangle_{1}|H\rangle_{2}$ representing the fully polarized component. This allows us to apply standard quantum techniques to measure the degree of polarization [2,3]. Also we can address the demonstration of the existence of the Zeno effect when monitoring the quantum-state purity of a two-dimensional system or the degree of polarization of a single photon. This is illustrated by two simple examples whose possible real physical realizations are outlined.

\section{THE SYSTEM}

Let us consider a single photon with transversal polarization. It is known that the most general state of polarization for the photon can be represented by the density matrix

$$
\rho=\frac{1}{2}(I+\boldsymbol{s} \cdot \boldsymbol{\sigma})
$$

where $I$ is the $2 \times 2$ identity matrix, $s=\operatorname{tr}(\rho \sigma)$ are the Stokes parameters $\left(s_{1}, s_{2}, s_{3}\right)$, with $|\boldsymbol{s}| \leqslant 1$, and $\boldsymbol{\sigma}$ are the three Pauli matrices. This density matrix $\rho$ can be decomposed in polarized part and unpolarized part as

$$
\rho=p \rho_{p}+(1-p) \rho_{\text {up }},
$$

where $p=|s|$ is the degree of polarization, $\rho_{p}=|p\rangle\langle p|$ is a pure state $|\mathrm{p}\rangle$ representing a fully polarized component (this is the mean or most probable polarization state), and $\rho_{\text {up }}=I / 2$ is a mixed state representing the fully unpolarized component. Note that there is a complete equivalence between quantumstate purity and degree of polarization for a single photon. In fact, we obtain from Eq. (1),

$$
\operatorname{tr}\left(\rho^{2}\right)=\frac{1}{2}\left(1+p^{2}\right)
$$

and pure states $\left(\rho^{2}=\rho\right)$ have the maximum degree of polarization $p=1$.

There is also a complete equivalence between the quantum density matrix $\rho$ and the normalized cross-spectral density function $\Gamma / \operatorname{tr} \Gamma$ of a classical light beam with the same Stokes 
parameters

$$
\Gamma=\left(\begin{array}{cc}
\left\langle\left|E_{x}\right|^{2}\right\rangle & \left\langle E_{x} E_{y}^{*}\right\rangle \\
\left\langle E_{x}^{*} E_{y}\right\rangle & \left\langle\left|E_{y}\right|^{2}\right\rangle
\end{array}\right)
$$

where $E_{x, y}$ are the corresponding electric-field components. In this regard, the decomposition of $\rho$ into polarized and unpolarized components in Eq. (2) mimics the same decomposition of $\Gamma$ in classical optics, leading to the degree of polarization $p=|s|$ as the relative weights of both components, the polarized and unpolarized light.

\section{A. Embedding in a doubled space}

In order to properly describe the evolution and observation of the degree of polarization, let us consider system realizations $\Phi \in H_{s} \otimes H_{s}$, where the photons are considered in pairs and the density matrix $\Phi$ mimics the decomposition (2),

$$
\Phi=p \Phi_{p}+(1-p) \Phi_{\mathrm{up}}
$$

having the following properties:

(i) $\Phi$ must lead to the correct reduced-density matrices for each photon when tracing over the other one,

$$
\rho_{1}=\operatorname{tr}_{2} \Phi, \quad \rho_{2}=\operatorname{tr}_{1} \Phi,
$$

with both $\rho_{1,2}=\rho$ given by the same density matrix (1), where $\operatorname{tr}_{j}$ represents trace over the photon $j$.

(ii) $\Phi_{p}$ and $\Phi_{\text {up }}$ represent the polarized and unpolarized components, respectively, in Eq. (2),

$$
\operatorname{tr}_{j} \Phi_{p}=\rho_{p}, \quad \operatorname{tr}_{j} \Phi_{\text {up }}=\rho_{\text {up }},
$$

for $j=1,2$, with the state $\Phi_{\text {up }}$ being independent of $\rho_{p}$, i.e., of the mean or most probable polarization.

(iii) We take full advantage of the enlarged space to impose orthogonality between the polarized $\Phi_{p}$ and unpolarized $\Phi_{\text {up }}$ components,

$$
\operatorname{tr}\left(\Phi_{p} \Phi_{\text {up }}\right)=0
$$

so that they are perfectly distinguishable (orthogonality between $\rho_{p}$ and $\rho_{\text {up }}$ is not possible in the single-photon space $\left.H_{S}\right)$.

The only solution for $\Phi$ satisfying all the above requirements is

$$
\begin{gathered}
\Phi_{p}=|\mathrm{P}\rangle\langle\mathrm{P}|, \quad| \mathrm{P}\rangle=|\mathrm{p}\rangle_{1}|\mathrm{p}\rangle_{2}, \\
\Phi_{\text {up }}=|\mathrm{U}\rangle\langle\mathrm{U}|, \quad| \mathrm{U}\rangle=\frac{1}{\sqrt{2}}\left(|H\rangle_{1}|V\rangle_{2}-|V\rangle_{1}|H\rangle_{2}\right),
\end{gathered}
$$

with $|\mathrm{p}\rangle_{1}|\mathrm{p}\rangle_{2}$ being $|\mathrm{V}\rangle_{1}|\mathrm{~V}\rangle_{2}$ or $|\mathrm{H}\rangle_{1}|\mathrm{H}\rangle_{2}$. The solution for $\Phi_{p}$ follows from condition (ii) as can be seen by expressing $\Phi_{p}$ in diagonal form and taking into account the fact that $\rho_{p}$ is pure. Then, the solution for $\Phi_{\text {up }}$ follows from condition (iii) because the states $|\mathrm{P}\rangle=|\mathrm{p}\rangle_{1}|\mathrm{p}\rangle_{2}$ are the set of $\mathrm{SU}(2)$ coherent states for total spin 1, that provides a resolution of identity for the subspace of symmetric states [4]. More specifically, expressing the most general $|\mathrm{p}\rangle$ as $|\mathrm{p}\rangle=\cos (\theta / 2)|H\rangle+\sin (\theta / 2) \exp (-i \phi)|V\rangle$ we get that
$|\mathrm{P}\rangle$ states are the $\mathrm{SU}(2)$ coherent states $|\mathrm{P}\rangle=|\Omega\rangle$,

$$
|\Omega\rangle=\sum_{m=-j}^{j}\left(\begin{array}{c}
2 j \\
j+m
\end{array}\right)^{1 / 2} \sin ^{j+m} \frac{\theta}{2} \cos ^{j-m} \frac{\theta}{2} e^{-i(j+m) \phi}|j, m\rangle,
$$

for $j=1$. In this case the $|j, m\rangle$ states are

$$
\begin{aligned}
|1,-1\rangle & =|H\rangle_{1}|H\rangle_{2}, \quad|1,1\rangle=|V\rangle_{1}|V\rangle_{2}, \\
|1,0\rangle & =\frac{1}{\sqrt{2}}\left(|H\rangle_{1}|V\rangle_{2}+|V\rangle_{1}|H\rangle_{2}\right),
\end{aligned}
$$

satisfying

$$
\int_{0}^{2 \pi} d \phi \int_{0}^{\pi} d \theta \sin \theta|\Omega\rangle\left\langle\Omega\left|\propto \sum_{m=-j}^{j}\right| j, m\right\rangle\langle j, m| .
$$

Thus, the only state orthogonal to all $|\mathrm{P}\rangle$ is the antisymmetric state $|\mathrm{U}\rangle$ with total spin 0 .

Moreover, note that $|\mathrm{U}\rangle$ is the only pure state invariant under any polarization transformation performed identically in both photons

$$
\exp \left[i \boldsymbol{u} \cdot\left(\boldsymbol{\sigma}_{1}+\boldsymbol{\sigma}_{2}\right)\right]|\mathrm{U}\rangle=|\mathrm{U}\rangle,
$$

where $\boldsymbol{u}$ is any real vector. The invariance of unpolarized light under any polarization-changing transformations is very much celebrated and becomes the definition of unpolarized light [5]. Thus the final form for the embedding $\Phi$ is

$$
\Phi=p|\mathrm{P}\rangle\langle\mathrm{P}|+(1-p)| \mathrm{U}\rangle\langle\mathrm{U}| .
$$

Other alternatives to Eq. (15) in terms of the coherent superposition of pure states are not useful since it cannot be granted that the degree of polarization will coincide with the probability of the fully polarized part.

In the most general case, the state (15) can be generated in practice by the alternative random generation of the states $|\mathrm{P}\rangle$ and $|\mathrm{U}\rangle$ with probabilities $p$ and $1-p$ respectively. In turn, the states $|\mathrm{P}\rangle$ and $|\mathrm{U}\rangle$ can be the outputs of nondegenerate type I and type II spontaneous parametric down-conversion, respectively.

The key advantage in the form (15) is that embedding $\rho_{p}$ and $\rho_{\text {up }}$ in the enlarged space leads to pure and orthogonal states so the degree of polarization can be observed as a standard quantum operator, with proper reduced states. This is crucial for the version of the Zeno effect addressed later.

\section{MEASUREMENT OF THE DEGREE OF POLARIZATION}

Since the fully polarized $|\mathrm{P}\rangle$ and unpolarized $|\mathrm{U}\rangle$ components in Eq. (15) are orthogonal, they are perfectly distinguishable by the measurement of a suitable operator. This measurement has been already performed experimentally by detecting whether the system is in the state $|\mathrm{U}\rangle$ [3]. It is known that nonlinear processes such as parametric down-conversion can produce the state $|\mathrm{U}\rangle$ from vacuum. The basic idea in Ref. [3] is to reverse the process: The vacuum state can be obtained at the output of a suitably arranged nonlinear process if and only if the input state is $|\mathrm{U}\rangle$.

Such a measurement, performed and reported in Ref. [3], has just two outputs that we may refer to as yes (the vacuum 
state is obtained) or not (the vacuum state is not obtained). In reference to Eq. (15), the probability of the output yes is given by the probability that the two photons are in the unpolarized state $|\mathrm{U}\rangle$, i.e., $\langle\mathrm{U}|\Phi| \mathrm{U}\rangle$, that readily gives the degree of polarization as

$$
p=1-\langle\mathrm{U}|\Phi| \mathrm{U}\rangle \text {. }
$$

According to the standard theory of ideal measurements, the associated reduced state is the fully unpolarized state $|\mathrm{U}\rangle$. The output not occurs with probability $p$, reducing the system to the fully polarized state $|\mathrm{P}\rangle$. Therefore, this measurement works exactly as desired for an ideal quantum observation of the polarized-unpolarized light decomposition. The degree of polarization then emerges as the probability that the photon is found in a fully polarized state.

If the dominant polarization state $|\mathrm{P}\rangle$ is known, we can measure the degree of polarization by projecting $\Phi$ on $|\mathrm{P}\rangle$, that in general is far much simpler than projecting on $|\mathrm{U}\rangle$. For example, if $|\mathrm{P}\rangle=|V\rangle_{1}|V\rangle_{2}$, we can place two ideal vertical polarizers just before two detectors that will detect coincidences when the input state is $|\mathrm{P}\rangle$ (both photons go through the polarizers) and no coincidences at all when the input state is $|\mathrm{U}\rangle$ (one of the photons is always stopped by the polarizers).

\section{ZENO EFFECTS WHEN OBSERVING THE EVOLUTION OF THE DEGREE OF POLARIZATION}

Let us use the above embedding and measurement to prove as simply as possible the existence of the Zeno-effect version addressed in this work. To this end we focus on the most simple and meaningful examples where the field state is initially fully polarized or fully unpolarized.

\section{A. Zeno effect on polarizing transformations}

Let us consider that initially we have fully unpolarized light $|\mathrm{U}\rangle$ experiencing a suitable transformation $T_{\theta}$ increasing the degree of polarization as $p_{\theta}=\sin ^{2} \theta$, where $\theta$ is the transformation parameter, with $\theta=0$ corresponding to the identity and $\theta=\pi / 2$ to full polarization. Next consider that the transformation is split into the product of $N$ identical consecutive transformations $T_{N}$ with parameter $\theta_{N}=\pi /(2 N)$ and $T_{N}^{N}=T_{\pi / 2}$, observing the degree of polarization after each step $T_{N}$ by projection on $|\mathrm{U}\rangle$. After the first transformation $T_{N}$ the system is found unpolarized with probability $\left\langle\mathrm{U}\left|T_{N}^{\dagger} \Phi T_{N}\right| \mathrm{U}\right\rangle=1-p_{N}=\cos ^{2} \theta_{N}$, the system being reduced to the initial state $|\mathrm{U}\rangle$. The probability that after the $N$ consecutive transformations and measurements the system remains unpolarized is

$$
\cos ^{2 N}\left(\frac{\pi}{2 N}\right), \text { with } \lim _{N \rightarrow \infty} \cos ^{2 N}\left(\frac{\pi}{2 N}\right) \rightarrow 1,
$$

that is, the Zeno effect occurs: The observation of the degree of polarization prevents light polarization.

In a suitable real physical realization, an initial state $|\mathrm{U}\rangle$ can be experimentally generated via type II spontaneous parametric down-conversion. The polarizing transformation can be implemented by placing two identical, less-than-perfect polarizers with a field-amplitude transmission coefficient $\cos \theta$ for the horizontal component and unit transmission coefficient for the vertical one, so horizontally polarized photons are removed with probability $\sin ^{2} \theta$. After the first split transformation $T_{N}$ with parameter $\theta_{N}=\pi /(2 N)$, the system is projected on $|\mathrm{U}\rangle$ (as reported in Ref. [3]) and found in the initial unpolarized state $|\mathrm{U}\rangle$ with probability $\cos ^{2}[\pi /(2 N)]$, which is the probability that no photon has been removed. Therefore, this scheme properly reproduces the desired evolution and observation of degree of polarization leading to the Zeno effect.

\section{B. Zeno effect on depolarizing transformations}

Let us now consider that initially we have fully polarized light $|\mathrm{P}\rangle$ and a transformation decreasing the degree of polarization as $p_{\theta}=\cos ^{2} \theta$, where $\theta$ is the transformation parameter reaching complete depolarization for $\theta=\pi / 2$. The transformation is again split into $N$ steps of parameter $\theta=$ $\pi /(2 N)$, measuring the degree of polarization after each step. In this case, simpler expressions are obtained by projecting on $|\mathrm{P}\rangle$ instead of $|\mathrm{U}\rangle$. After the first step, the probability that the state remains polarized is $\left\langle\mathrm{P}\left|T_{N}^{\dagger} \Phi T_{N}\right| \mathrm{P}\right\rangle=p_{N}=\cos ^{2} \theta_{N}$, with the system being reduced to the initial state $|\mathrm{P}\rangle$. The probability that all the $N$ consecutive measurements confirm that the state is polarized is the same as in Eq. (17). The photon tends to remain fully polarized, so the Zeno effect holds: The observation of the degree of polarization prevents light depolarization.

In a simple real physical realization, the initially fully polarized state, say $|\mathrm{P}\rangle=|V\rangle_{1}|V\rangle_{2}$, can be generated in experiment via nondegenerate type I spontaneous parametric down-conversion. The depolarizing transformation can be implemented by placing two polarization rotators (via Faraday effect or half-wave plates) producing opposite linear polarization rotation of angles $\theta$ and $-\theta$ in each mode, giving

$$
\begin{aligned}
T_{\theta}|V\rangle_{1}|V\rangle_{2}= & \cos ^{2} \theta|V\rangle_{1}|V\rangle_{2}-\sin ^{2} \theta|H\rangle_{1}|H\rangle_{2} \\
& +\sin \theta \cos \theta\left(|H\rangle_{1}|V\rangle_{2}-|V\rangle_{1}|H\rangle_{2}\right) .
\end{aligned}
$$

In the case of the single-step split transformation with sufficiently small parameter $\theta_{N} \propto 1 / N \ll 1$ (the case of interest for the Zeno effect), the transformed state can be approximated to first order as

$$
T_{N}|\mathrm{P}\rangle \simeq|\mathrm{P}\rangle+\sqrt{2} \theta_{N}|\mathrm{U}\rangle .
$$

Thus, the probability that all the $N$ consecutive measurements confirm that the state is actually $|\mathrm{P}\rangle$ is $p_{N}^{N} \simeq\left(1-2 \theta_{N}^{2}\right)^{N} \simeq$ $\exp \left(-2 N \theta_{N}^{2}\right)$ with $p_{N}^{N} \rightarrow 1$ as $N \rightarrow \infty$, and hence, the Zeno effect holds.

\section{CONCLUSIONS}

We have examined a version of the Zeno effect for the observation of quantum-state purity with some striking features. These are that the monitored physical property cannot be represented by a standard operator in the single system space and the evolution prevented by observation is not unitary.

The key point of the analysis is that by suitably embedding the system in an enlarged space, pure and fully mixed components lead to orthogonal subspaces. In addition, by 
imposing desirable properties, we show that the system representation in the enlarged space is unique. The degree of purity can then be observed as an standard quantum operator, including proper reduced states. This is crucial for the version of the Zeno effect addressed here, providing a simple proof of principle demonstration of the effect. Besides, we have illustrated this possibility with two feasible physical realizations.

We emphasize that a rather abstract property such as quantum-state purity becomes a directly measurable quantity via light polarization and photon entanglement. Since purity is quadratic in the density matrix, $\langle\rho\rangle=\operatorname{tr}\left(\rho^{2}\right)$, we may say that the monitored observable and the observed system coincide and that the system observes itself. This recalls the Greek myth of Narcissus, whose vital (nonunitary) evolution ended in similar circumstances of stopped dynamics.

\section{ACKNOWLEDGMENTS}

A.L. acknowledges support from Projects No. FIS201235583 of the Spanish Dirección General de Investigación del Ministerio de Economía y Competitividad and QUITEMAD No. S2009-ESP-1594 of the Consejería de Educación de la Comunidad de Madrid. I.G. and M.A.P. acknowledge support from Project No. FIS2010-22082 of the Spanish Ministerio de Economía y Competitividad. M.A.P. acknowledges support from Project No. MTM2012-39101-C02-01 of the Spanish Ministerio de Economía y Competitividad.
[1] B. Misra and E. C. G. Sudarshan, J. Math. Phys. 18, 756 (1977); L. A. Khalfin, Zh. Eksp. Teor. Fiz. 33, 1371 (1957); Sov. Phys. JETP 6, 1053 (1958); R. G. Winter, Phys. Rev. 123, 1503 (1961); W. Yourgrau, in Problems in the Philosophy of Science, edited by I. Lakatos and A. Musgrave (North-Holland, Amsterdam, The Netherlands, 1968), pp. 191 and 192; W. M. Itano, D. J. Heinzen, J. J. Bollinger, and D. J. Wineland, Phys. Rev. A 41, 2295 (1990).
[2] N. Gisin, J. Mod. Opt. 48, 1397 (2001).

[3] M. Legré, M. Wegmüller, and N. Gisin, Phys. Rev. Lett. 91, 167902 (2003).

[4] F. T. Arecchi, E. Courtens, R. Gilmore, and H. Thomas, Phys. Rev. A 6, 2211 (1972).

[5] G. S. Agarwal, Lett. Nuovo Cimento Soc. Ital. Fis. 1, 53 (1971); H. Prakash and N. Chandra, Phys. Rev. A 4, 796 (1971) 